\title{
LA HISTORIA CLÍNICA EN SALUD MENTAL
}

\section{Sergio Ramos Pozón ${ }^{1}$}

Resumen: En este artículo analizamos algunas cuestiones de la historia clínica cuando se enfoca al ámbito de la salud mental. En primer lugar, definimos qué es, qué contenido ha de tener y cómo estructurarla. En segundo término, exponemos algunos principios deontológicos, unos principios éticos y unas virtudes éticas para guiar la elaboración de la HC y el manejo de su información. En tercero, se examina a quién pertenece y quién puede acceder a la información. Por último, se exponen datos empíricos sobre la opinión de los pacientes y los profesionales sobre los registros médicos.

Palabras clave: historia clínica, salud mental, estructura, confidencialidad, bioética

\section{The medical record in mental health}

Abstract: In this paper we'll address the issue of medical record in mental health. We structure this paper in four sections. First, we define what is the medical record, which is their content, and how it has to be structured. Second, we expose some deontological principles, ethical principles and ethical virtues to guide the development of the medical record and information management. Third, we analyze the question of who owns the medical record and who can access the information. Finally, we expose some empirical data about the opinion of both patients and professionals about the medical record.

Key words: medical records, mental health, structure, confidentiality, and bioethics

\section{A história clínica em saúde mental}

Resumo: Neste artigo analisamos algumas questôes da história clínica quando se enfoca o âmbito da saúde mental. Em primeiro lugar, definimos o que é, qual conteúdo deve ter e como estruturá-la. Em segundo termo, expomos alguns princípios deontológicos, alguns princípios éticos e algumas virtudes éticas para guiar a elaboração da HC e o manejo de sua informação. Em terceiro, se examina a quem pertence e quem pode acessar a informação. Por último, se expóem dados empíricos sobre a opinião dos pacientes e dos profissionais sobre os registros médicos.

Palavras-chave: história clínica, saúde mental, estrutura, confidencialidade, bioética

\footnotetext{
${ }^{1}$ Universidad de Barcelona, Espańa

Correspondencia: ramospozon@hotmail.com
} 
Para la elaboración de este trabajo, nos basamos en la revisión de la normativa jurídica que regula la historia clínica (HC) en España. Junto a ese análisis, se lleva a cabo una reflexión sobre los principios éticos que subyacen en la HC, así como las virtudes necesarias de los profesionales durante la recogida de la información para conseguir así un mayor respeto por la persona. Esa exposición se acompańa de datos empíricos sobre la opinión de pacientes y profesionales.

\section{La historia clínica: definición y estructura}

La HC es el conjunto de datos biopsicosociales vinculados a la salud de un paciente. Tiene como fin la asistencia sanitaria, aunque también tiene usos de carácter judicial, epidemiológico, de salud pública, de investigación y de docencia. La Ley $41 / 2002$, de 14 de noviembre, dedica el capítulo $\mathrm{V}$ a la HC. En el artículo 14 la define como "el conjunto relativo a los procesos asistenciales de cada paciente, con la identificación de los médicos y de los demás profesionales que han intervenido en ello, con objeto de obtener la máxima integración posible de la documentación clínica de cada paciente, al menos, en el ámbito de cada centro".

Esta ley también contempla qué contenido ha de tener la HC. En el artículo 15 se constata la necesidad de anotar toda aquella información que se considere, a criterio médico, necesaria para el conocimiento veraz y actualizado del estado de salud del paciente. En particular, ha de contener: 1) el documento relativo a la hoja clínica-asistencial; 2) la autorización del ingreso; 3) el informe de urgencia; 4) la anamnesis y exploración física; 5) la evolución; 6) las órdenes médicas; 7) la hoja de interconsulta; 8) los informes de exploración complementaria; 9) el consentimiento informado; 10) el informe de anestesia; 11) el informe de quirófano o de registro de parto; 12) el informe patológico; 13) la evolución y planificación de cuidados de enfermería; 14) la aplicación terapéutica de enfermería; 15) el gráfico de constantes; 16) el informe clínico de alta.

El contenido de la HC prácticamente está centrado en el ámbito médico en general, y apenas se hace referencia a otros contextos de carácter psicosocial, olvidándose que en la salud de una persona intervienen aspectos biopsicosociales y no solo biológicos. Y si hay poca alusión a esos aspectos, menos aún a los problemas específicos de salud mental(1). En psiquiatría se quiere restablecer la salud del paciente, por eso no solo hay que reparar un órgano, sino también aliviar el sufrimiento psicológico y abordar los déficit sociales, cognitivos, etc. Además, en salud mental intervienen muchos profesionales — psicólogos, psiquiatras, trabajadores sociales, etc. - y cada uno aporta una información distinta. Por tanto, la $\mathrm{HC}$ no puede estar centrada exclusivamente en los datos biológicos, sino que precisa mencionar otras cuestiones de carácter psicosocial.

Dado que estamos analizando una HC psiquiátrica con un carácter biopsicosocial, descartamos que la psiquiatría pueda ser exclusivamente biologicista. Como señala $\mathrm{Baca}(2: 215)$ se estructura de manera trifronte: 1) una psiquiatría de hechos, en la que el signo es la manifestación de la biología de las enfermedades mentales; 2) una psiquiatría que refleja la realidad de la identidad humana en el marco de las narrativas, la cual se expresa mediante sintomas en cuanto fenómenos, sea mediante actos de habla o de conducta, y 3 ) una psiquiatría que reflexiona sobre la implicación de los valores individuales y compartidos, tanto en la categorización de lo que son las alteraciones mentales, como del modo en que son vistos y enjuiciados por los afectados (cuidadores y sociedad en general). Y desde esta estructuración creemos que la HC ha de contener cuatro apartados: signos, síntomas, anotaciones subjetivas y cuestiones éti$\operatorname{cas}^{2}$. Y todo ello ha de estar centrado para y en el paciente.

\footnotetext{
${ }^{2}$ Cañas F., Santander F. y Morera B.(3:48), siguiendo a Appelbaum P. y Gutheil T., estructuran la HC psiquiátrica en: hechos (datos objetivos), juicios (pensamientos y razonamientos) y reflexiones (que implican un estilo narrativo). Además, hay dos tipos de notas: notas de progreso (similares a las $\mathrm{HC}$ convencionales, fundamentadas en datos objetivos y que son de acceso del paciente) y notas de proceso (constituidas por aspectos menos objetivos del sujeto, como son fantasías, emociones..., y de las interpretaciones y especulaciones del equipo de profesionales). Por su parte Gracia D.(4:142-147) señala una $H C$ por problemas. Considera que a la tradicional HC deberá insertar también apartados que recojan: 1) identificación de los problemas morales; 2) evaluación de la capacidad; 3) consentimiento informado que exprese la información que el paciente ha recibido; 4) otros documentos que indiquen los deseos del paciente, como puede ser el documento de voluntades anticipadas; 5) un apartado que controle y evalúe la calidad del consentimiento informado; 6) los diferentes cursos de acción posible; 7) evaluación de los problemas morales, y 8) el informe de alta. Nuestro planteamiento esta muy influenciado por esta $H C$ por problemas.
} 
En un primer momento se deben recoger aquellos datos que ayudan a detectar y tratar la situación clínica. El signo es una señal biológica, se detecta gracias a procedimientos exploratorios y puede o no acompañarse de sintomas y/o expresarse mediante ellos. El signo en medicina es "aquella señal o hecho que indica válida y fiablemente la existencia de una enfermedad y que, además, posee la suficiente sensibilidad y especificidad como para ser considerado marcador de estado de dicha situación patológica" (2:125). Se refiere al diagnóstico, a la etiopatogenia y al pronóstico de la enfermedad, pero también ayuda a realizar un plan terapéutico. En este apartado tienen cabida todos aquellos aspectos que seńala la Ley 41/2002 en su artículo 15, a excepción del consentimiento informado, que debe estar en el apartado de cuestiones éticas.

En segundo lugar se debe anotar aquellos sintomas que refleje el paciente. El sintoma remite a la conciencia del paciente que sufre, es decir, es la concreción de un sufrimiento, y se configura y cobra sentido a raíz de su biografía. Según Baca(2:191), se trata de "un acontecimiento vital configurador e inmediatamente configurado, que pone en marcha un proceso que no es posible inscribir en otro lugar que no sea una de las narrativas que configuran la narrativa del sujeto. Esta narrativa es la narrativa de la enfermedad en cuanto suceso existencial". Recordemos que en el ámbito psiquiátrico pueden darse síntomas de ansiedad, depresivos, obsesivos-compulsivos, maníacos, delirantes, alucinatorios, de afectación de la conciencia (desorientación y desorganización) y demenciales (2:183).

Por un lado, el paciente revela su sufrimiento mediante los síntomas al mostrar su estado anímico. También los síntomas exteriorizan cómo se manifiesta la enfermedad, o verbal (verbaliza su delirio, sus alucinaciones...) o conductualmente (apatía, abulia, etc.). Algunos síntomas, tales como los depresivos o los de ansiedad, reflejan cómo interactúa la persona con el ambiente familiar, comunitario... Por otro lado, el equipo de salud mental ha de estar atento a estos síntomas y anotar aquello que considere necesario para el abordaje de la patología. Son de gran ayuda preguntas como: ¿de qué modo sufre?, ¿por qué sufre? o ¿qué miedos tiene? Estas anotaciones son fruto de la observación objetiva de una situación clínica, por lo que no son juicios subjetivos. Se trata, por tanto, de la constatación de hechos, de descripciones clínicas propias de una enfermedad, de orientaciones clínicas, de diagnósticos, etc., que hacen alusión a un modo de praxis profesional narrativa, pues está basada en la biografía del paciente.

Un tercer apartado lo constituyen las anotaciones subjetivas. En el artículo 18.3 de la Ley 41/2002 se hace referencia a los derechos de acceso a la historia clínica y allí se menciona la posibilidad de dejar anotaciones subjetivas; sin embargo, no se aclara cuál es su significado. Aunque en España algunas comunidades autónomas sí disponen de normativa jurídica que las definen, la gran mayoría no lo hace ${ }^{3}$.

Las anotaciones subjetivas no se refieren a cuestiones de hechos, sino a

"opiniones del facultativo cuyo origen no es deducible objetivamente y que no surgen de la observación de un hecho biológico o de su evolución, ni plantean alternativas diagnósticas o decisiones clínicas, y que son sólo consideraciones personales anotadas como ayuda propia o como orientación"(5).

\footnotetext{
${ }^{3}$ En la comunidad autónoma de Extremadura, la Ley 3/2005, de 8 de julio, de información sanitaria y autonomía del paciente, art. 32.4.19d, las define como "impresiones de los profesionales sanitarios, basadas en la exclusiva percepción de aquellos, y que, en todo caso, carecen de trascendencia para el conocimiento veraz y actualizado del estado de salud del paciente, sin que puedan tener la consideración de un diagnóstico".

En Galicia, el Decreto 29/2009, de 5 de febrero, por el que se regula el uso y acceso a la historia clínica electrónica, art. 21, entiende por anotaciones subjetivas las "valoraciones personales, sustentadas o no en los datos clínicos de que se disponga en ese momento, que no formando parte de la historia clínica actual del/de la paciente o usuario/a, puedan influir en el diagnóstico y futuro tratamiento médico una vez constatadas".

Castilla la Mancha, en la Circular 1/2009, sobre uso, acceso, cesión de datos y conservación de la Historia Clínica en el ámbito del Sescam, en el apartado 4.3.7b indica que solo serán consideradas como tales las valoraciones sobre hipótesis diagnósticas no demostradas, suposición de incumplimiento terapéutico, presuposición de tomar otro tratamiento no declarado, sospechas de hábitos no reconocidos, sospecha de haber sido víctima de malos tratos, y comportamientos insólitos.

En el País Vasco, en el Decreto 38/2012, de 13 de marzo, sobre historia clínica y derechos y obligaciones de pacientes y profesionales de la salud en materia de documentación clínica, en el artículo 7.4: "Se entenderá por anotaciones subjetivas las impresiones o valoraciones personales de las y los profesionales sanitarios no sustentadas directamente en datos objetivos o pruebas complementarias y que, en su criterio, resulten de interés para la atención sanitaria de la persona paciente".
} 
Por último, un cuarto apartado debería destinarse a cuestiones éticas. Aunque se podrían especificar tantos aspectos como los profesionales de salud consideren oportuno, en el ámbito psiquiátrico es importante tener en cuenta una serie de temas susceptibles de conflictos, a saber: el tipo de relación asistencial(6), las decisiones por representación(7), la competencia del paciente, el consentimiento informado(8), el documento de voluntades anticipadas(9) o la calidad de vida del paciente(10). Si se desea un conocimiento veraz y actualizado de la salud del paciente, este ha de participar activamente en la $\mathrm{HC}$ para que haya más información sobre sí mismo. Esto también promueve un mayor protagonismo del paciente en las cuestiones vinculadas con su salud y la oportunidad de recibir más información sobre su proceso asistencial.

Durante la relación asistencial es frecuente que el psiquiatra se detenga en los miedos, expectativas, deseos, etc., en definitiva, en los "valores ocultos"(11), así como en sus relaciones de pareja, amistades, etc. También se abordarán cuestiones vinculadas con el estigma y la discriminación por tener una enfermedad mental. Los profesionales necesitan ser muy cautelosos con estos datos, por tratarse de datos sensibles. Es clave anotar quiénes son los cuidadores, familia, referentes, representantes, etc. En casos de decisiones por representación, es importante anotar quién ha sido el sustituto, qué criterio se sigue para decidir: criterio subjetivo, el criterio del juicio sustitutivo o el criterio del mejor interés(12:98-134), y anotar si ha habido conflicto de intereses. Se debe comprobar si el paciente tiene anotado un documento de voluntades anticipadas. Independientemente de quién sea el que tome la decisión, representante o paciente, es necesario recabar el consentimiento informado. Si los profesionales se aferran al privilegio terapéutico, decidiendo obviar algún tipo de información porque consideran que puede ser potencialmente dañina, se dejará constancia sobre qué datos y por qué motivos se ocultó.

Dado que un requisito del consentimiento informado es que el paciente sea competente para decidir por sí mismo, hay que anotar aquellas insuficiencias del paciente que le impidan decidir autónomamente.

\section{Derechos, obligaciones, virtudes y principios éticos}

El Grupo de trabajo de bioética de la semFYC(13) defiende unos principios deontológicos para garantizar el respeto de los datos sanitarios.

El principio de sobriedad (pertinencia) establece que los profesionales sanitarios deben recabar y anotar lo estrictamente necesario para una atención médica de calidad. Esto implica no tener que revelar, si no es imprescindible, datos confidenciales.

El principio de transparencia indica la necesidad de que el paciente sepa qué tipo de información hay en la $\mathrm{HC}$, así como quién y bajo qué condiciones los profesionales pueden tener acceso a ella.

El principio de responsabilidad conlleva que los profesionales han de ser cuidadosos con el manejo de la información y que el trabajo en equipo no debe utilizarse para difuminar responsabilidades.

El principio de protección universal sostiene que en todos los centros sanitarios y para todos los pacientes han de adoptarse unas medidas de seguridad que garanticen la protección de los datos sanitarios.

Estos principios recuerdan la necesidad de garantizar la confidencialidad de los datos sanitarios. La confidencialidad es tanto un derecho como una obligación.

Beauchamp y Childress(14:405-408) sostienen que la confidencialidad es necesaria porque si los pacientes no se fiasen de que los profesionales respetarán la confidencialidad, no aportarían información completa sobre su salud, y sin dicha información los profesionales serían incapaces de diagnosticar de manera precisa y/o recomendar tratamientos.

Pese a ello, la normativa jurídica marca unas excepciones que limitan el derecho a la confidencialidad. Se justifica la divulgación de información si es por un estado de necesidad que evite un mal propio o ajeno. Si se trata de cumplimiento de un deber: 1) de denunciar un delito; 2) de impedir un delito; 3) de testificar, y 4) de comunicar casos de enfermedades infecto-contagiosas(15:24). 
Gracia(16:315-318) considera dos tipos de hipótesis para blindar los datos y asegurar que se respete la información. La primera es la "teoría del blindaje débil del secreto médico", la cual afirma que la confidencialidad ha de ceder en caso de entrar en conflicto con otros derechos fundamentales. Es el juez quien determina en qué situaciones los profesionales pueden revelar información confidencial.

Se reconoce que el deber de confidencialidad puede quebrantarse si el paciente da el consentimiento para que se informe sobre los datos sanitarios que a él se refieren. En ese caso, la autonomía y la libertad individual prevalecen. Ahora bien, en casos de estado de necesidad o de cumplimiento del deber prevalecen los principios de beneficencia y la no-maleficencia, lo cual señala la necesidad de romper con la confidencialidad. Por otro lado, la "teoría del blindaje fuerte del secreto médico" comparte con la anterior el hecho de aceptar las excepciones al secreto médico; sin embargo, considera que estas han de ser marcadas por los profesionales y no por la normativa jurídica.

No obstante, por muchos mecanismos informáticos que garanticen la confidencialidad, así como leyes que marquen su obligatoriedad, en último término el deber de confidencialidad está en manos de los profesionales. Ellos deben tener unas cualidades para que haya un respeto por la persona y se asegure que no se revelará información. Tanto en la elaboración de la HC como en su deber de confidencialidad, los profesionales requieren de las virtudes de fidelidad, confianza, veracidad, honestidad, respetuosidad, etc.

Pero si el tema de la confidencialidad de los datos es un problema que genera gran debate, no es menos el que suscita a quién pertenece la HC y quién puede acceder a ella. En el ámbito de la salud mental esta última cuestión tiene mayor relevancia, porque intervienen múltiples profesionales de distintas formaciones y porque puede haber incompetencia para la toma de decisiones que indica que ha de informarse a otras personas sobre la situación clínica del paciente, y porque en salud mental la HC debe ser muy compartida.

\section{Propiedad y acceso de la HC}

Un tema frecuente en la literatura sobre la $\mathrm{HC}$ es a quién pertenece dicho documento. Los problemas derivan de que el documento es elaborado por un profesional, o un conjunto de profesionales, cuyo contenido son datos del paciente, aunque con anotaciones subjetivas de los profesionales(17:112-119). Pese al posible debate, la Ley 41/2002, art. 5, sostiene que el titular de la HC es el paciente. La regulación de los datos vinculados al ámbito de la salud queda recogido en la Ley 41/2002, por la Ley Orgánica 15/1999, de 13 de diciembre, de Protección de Datos de Carácter Personal, y por el Real Decreto 1720/2007, de 21 de diciembre, por el que se aprueba el Reglamento de desarrollo de la Ley Orgánica 15/1999, de 13 de diciembre, de protección de datos de carácter personal.

Creemos que una buena solución al respecto es la del Comité de Bioética de Catalunya(18) al analizar la cuestión no sobre quién es el propietario, sino cuáles son los derechos y obligaciones de cada uno. De modo que los profesionales tienen el derecho y el deber de realizar la HC, la Administración el deber de custodia, de seguridad y de igualdad, y el paciente tiene derechos esenciales sobre los datos que la integran.

Pero una cosa es quién es el propietario o qué derechos y obligaciones tiene respecto de la $\mathrm{HC}$, y otra quiénes pueden acceder a ella. A efectos del art. 18, tienen derecho de acceso el paciente, el tutor o representante, los profesionales y los familiares. Ahora bien, caben matizaciones sobre esta accesibilidad a la información.

Según la ley, el paciente es quien tiene derecho a acceder a su HC y a obtener una copia de la misma. Pero este acceso está limitado en los siguientes casos(18:18-19): 1) cuando el tratamiento está en múltiples bases de datos, el responsable del fichero puede pedir que la persona solicitante especifique qué tipo de fichero desea; 2) se trata de un ejercicio anual, ya que la persona no podrá ejercer tal derecho más de una vez por año; 3) no podrá ejercerse tal derecho si es en perjuicio del derecho de terceras personas a la confidencialidad, ni en detrimento del derecho de los profesionales que contribuyen a su elaboración, que pueden apelar 
al derecho de reserva de sus anotaciones subjetivas, y 4) en supuestos en los que una ley o norma de derecho así lo prevea, como es el caso de la necesidad terapéutica, en el que, según el juicio del profesional, la revelación de la información pueda suponer un grave riesgo para la salud del paciente.

Como señala Fernández(17:119-128), determinados datos de la $\mathrm{HC}$ pueden ser perjudiciales para el paciente o ser malinterpretados, pues la $\mathrm{HC}$ suele estar redactada con tecnicismos. Pese a que el paciente tenga derecho de acceso, no por ello se tiene que entregar toda la documentación. Por un lado, se ha de excluir información relativa a otras personas. Por otro, no tienen por qué entregarse las anotaciones subjetivas. La entrega de las mismas no solo no ayuda al paciente, sino que podría suponer falsas ideas y además coartaría la libertad de los profesionales para escribirlas. Y estos aspectos en un paciente con esquizofrenia es especialmente pertinente recordar.

Un problema sobre las anotaciones subjetivas es si deben considerarse un derecho o un privilegio de los profesionales (19). Consideramos que merecen un tratamiento confidencial, aunque no por ello han de ser consideradas un derecho de los profesionales. Y si no cumplen con la finalidad de la $\mathrm{HC}$, carece de sentido incorporarlas en la misma. Ahora bien, si cumplen con ello y no hay otro medio para constar tal información, y si a criterio médico esta puede ocasionar empeoramiento grave del paciente, se debe admitir la reserva de estas anotaciones.

Un ejemplo en psiquiatría es la sospecha de que el paciente manifiesta algún tipo de cuadro delirante que, aunque requiere de un mayor análisis, puede ser una psicosis incipiente. En el caso de que este tuviese acceso a su HC, este dato podría dificultar la relación asistencial al sentirse más vigilado o incluso pudiendo potenciar más el delirio y/o alucinación.

El derecho de acceso también puede darse mediante representación debidamente acreditada. Hemos visto que, en pacientes con esquizofrenia, cuando hay problemas con la competencia se requerirá un tutor o representante. Si es declarado incapaz legalmente será esta figura representativa la que tenga derecho de acceso. La familia solo puede acceder a la HC si presenta una acreditación y justificación del propio paciente o si el paciente ha fallecido y no ha dejado constancia de su negativa. En ningún caso se entregará información que haga alusión a su intimidad, que perjudique a terceras personas y/o las anotaciones subjetivas de los profesionales.

Es lógico que los profesionales que intervienen en los procesos terapéuticos y diagnósticos también tengan derecho de acceso. En salud mental intervienen muchos profesionales de distintas ramas, a saber, médico de atención primaria, psicólogo, psiquiatra, enfermeros, trabajadores sociales, profesionales de gestión y servicios informáticos, entre otros. Cada uno puede aportar información relevante dentro de su propio campo. Y aquí puede haber problemas de confidencialidad.

El Comité de Bioética de Catalunya(20) cree que sería positivo que hubiese un registro de información destinada a un profesional o un equipo especializado, y que si otros profesionales quisieran acceder deberían pedir el consentimiento del paciente y siempre dejar constancia de quién entra.

Este planteamiento queda recogido en el artículo 13 del Decreto 38/2012, de 13 de marzo, sobre historia clínica y derechos y obligaciones de pacientes y profesionales de la salud en materia de documentación clínica. Se establece que el acceso a la información será selectivo en consideración a la categoría profesional, al tipo de datos y al lugar o puesto de trabajo en relación al proceso asistencial. Al acceder a la información, el profesional ha de identificarse de manera inequívoca. Si se trata de profesionales de otros centros o servicios sanitarios diferentes, se necesitará una justificación explícita sobre los fines asistenciales.

Dado que principalmente los que consultan la HC son los pacientes y los profesionales, es importante conocer la opinión de estos sobre la información y el modo en el que se anota. Y es importante conocerla para contribuir a la mejora de la redacción de la HC y al manejo de la información. A continuación examinamos algunos estudios que evalúan cuantitativa y cualitativamente sus opiniones. 


\section{Opinión de los pacientes y los profesionales} sobre la HC

Existen pocos estudios que evalúen la opinión de los pacientes y profesionales sobre la HC. Esto puede deberse a que hace relativamente poco la HC ha sido informatizada, y que a raíz de esto se ha generado un gran debate alrededor de la confidencialidad.

Perera G., Holbrook A. et al.(21) examinan la opinión de 490 pacientes y 46 profesionales sobre los beneficios y perjuicios de la HC informatizada. La gran mayoría de pacientes $(58 \%)$ y de profesionales $(70 \%)$ creen que hay más beneficio que riesgos para la confidencialidad al informatizar la HC. Además, consideran positivo compartir la información entre el círculo de profesiones destinados al cuidado del paciente, mientras que tienen reticencias cuando se trata de otros profesionales. Resulta significativo que los pacientes valoran positivamente $(60 \%)$ poder tener una copia de toda su información, mientras que la opinión de los profesionales está dividida: un 33\% lo ve muy favorable frente a un $39 \%$ que muestra un desacuerdo total.

Salomon R., Urbano J., et al.(22) entrevistan a 56 psiquiatras para saber sus opiniones respecto al uso de la HC. Todos sostienen que la confidencialidad de los datos es una cuestión muy importante, por lo que es positivo utilizar estrictos controles de seguridad. La mayoría de ellos no piensa que mantener los datos referentes al ámbito psiquiátrico separado de la información médica general empeore el estigma hacia estas personas; sin embargo, no creen que el estigma se reduciría si la información psiquiátrica estuviese en la misma ubicación que en la general. Aunque puede preocupar a los pacientes que otros profesionales puedan acceder a su información psiquiátrica, los encuestados no percibieron que sus pacientes tuvieran un cambio de conducta al usar la HC informatizada. Por último, los psiquiatras reconocen que deberían dedicar más tiempo a rellenar la $\mathrm{HC}$, en particular sobre los efectos adversos del tratamiento farmacológico.
Fidel S., Eiroa F., et al.(23) recogen la opinión de 58 profesionales sobre la confidencialidad en la HC y buscan identificar las estrategias utilizadas para preservarla. La mayoría de los encuestados comparten la idea de que la información en salud mental requiere más confidencialidad que en otras especialidades. Manifiestan que algunos pacientes se han sentido preocupados porque otros profesionales puedan acceder a su información psiquiátrica e incluso algunos solicitan abiertamente que no se anote en la HC ciertos datos. Por eso, algunos profesionales reconocen no anotar algún tipo de información, como episodios violentos, agresiones, sospechas, aspectos de identidad sexual, etc., y optan por recogerlas en la pestaña de "anotaciones subjetivas". Para salvaguardar la confidencialidad, proponen fortalecer la seguridad del sistema, establecer limitaciones de acceso a otros ámbitos no propiamente psiquiátricos, solicitar el consentimiento informado al paciente, entre otros.

Aunque sería necesario realizar más estudios sobre la percepción de los profesionales y los pacientes ${ }^{4}$, sí que podemos extraer algunas conclusiones.

Es pertinente construir cuestionarios que evalúen el uso y la percepción de la HC en el ámbito psiquiátrico, pues en este medio es mejor la preocupación por el uso que se pueda hacer de la información ${ }^{5}$. La confidencialidad adquiere un mayor este papel más preponderante, pues en salud mental interviene un equipo multidisciplinar de profesionales y hay una mayor preocupación por los datos sensibles y las anotaciones subjetivas. El paciente tiene derecho a detallar qué información desea que otros profesionales conozcan y/o cuál quiere eliminar o no dejar reflejada, pero los profesionales deben valorar el nivel de competencia del paciente para asumir el riesgo, que se implique al mismo paciente, así como el riesgo de atentar contra la lex artis y buenas prácticas en salud mental.

\footnotetext{
${ }^{4}$ Es interesante el estudio de Iraburu M, Chamorro J, De Pedro M.(24), que analiza conocimientos, comportamientos y opiniones de médicos y enfermeros sobre el tema de la confidencialidad; y en el marco de la salud mental, el estudio de Boyer L, Renaud M, et al.(25) sobre la percepción de los profesionales respecto de la confidencialidad.

${ }^{5}$ Puede servir como ejemplo el cuestionario de Boyer L, Baumstrarck $\mathrm{K}$, et al.(26).
} 


\section{Resultados}

El contenido de la HC prácticamente está centrado en el ámbito médico en general, olvidándose de que en la salud de una persona intervienen aspectos biopsicosociales y no solo biológicos. Esto va a significar la estructuración de la HC en cuatro apartados: signos, sintomas, anotaciones subjetivas y cuestiones éticas. Las cuestiones éticas (principios deontológicos, principios éticos y virtudes éticas) indican la necesidad de prestar mucha atención a los datos sensibles recogidos en la $\mathrm{HC}$, para garantizar el respeto por la confidencialidad, que ha de ser entendida tanto un derecho como una obligación. El análisis de ese derecho y esa obligación por salvaguardar la confidencialidad de los datos nos hizo replantearnos cuestiones referentes a la propiedad y al acceso de la HC. En particular, examinamos qué obligaciones y derechos hay inmiscuidos, quién puede acceder a la HC y bajo qué situaciones pueden acceder otros profesionales. Por último, hay que evaluar la opinión de los profesionales y los pacientes sobre la HC, pues son los principales afectados por las decisiones. La confidencialidad adquiere un mayor papel, pues en salud mental interviene un equipo multidisciplinar de profesionales y hay una mayor preocupación por los datos sensibles y las anotaciones subjetivas. El paciente tiene derecho a concretar qué información desea que otros profesionales conozcan y/o cuál quiere eliminar o no dejar reflejada, pero los profesionales deben valorar el nivel de competencia del paciente para asumir el riesgo, pero siempre teniendo en cuenta no atentar contra la lex artis y buenas prácticas en salud mental.

\section{Discusión}

En este artículo hemos analizado varias cuestiones referentes a la HC. Por HC entendemos el conjunto de datos referentes a la salud del paciente y cuyo objetivo principal es la asistencia sanitaria, aunque también la normativa jurídica acepta otros tipos de propósitos. En cuanto a su contenido, hemos constatado que ha de ser biopsicosocial y su estructuración ha de ser mediante cuatro apartados, a saber: signos, síntomas, anotaciones subjetivas y cuestiones éticas.
Para analizar con más rigor la $\mathrm{HC}$, se ha constatado la necesidad de que esté fundamentada en una serie de principios deontológicos, normas morales y principios éticos. La confidencialidad ha sido un punto importante, manifestándose como un derecho del paciente así como del profesional, aunque también como una obligación legal hacia el equipo sanitario. Aunque varios argumentos son los que fundamentan la necesidad de respetar la confidencialidad, la normativa jurídica establece algunas excepciones que justifican la divulgación de información: estado de necesidad y el cumplimiento del deber. Y es que tanto el deber de respetar la información como sus posibles excepciones están fundamentadas en principios y virtudes éticas.

También se ha analizado el problema de la propiedad y acceso a la HC. La ley estipula que es el paciente el titular de la HC. Los profesionales, la Administración y el paciente tienen derechos y obligaciones. Varias son las personas que tienen derecho al acceso a la información, aunque en todos los casos se ha de respetar el derecho a la intimidad de terceras personas y de los profesionales.

Por último, puesto que deseamos una mejora en su redacción y un mejor manejo de la información sanitaria, hemos analizado algunos estudios que evalúan cuál es la opinión de los pacientes y los profesionales sobre la HC.

De todo ello podemos extraer unas conclusiones. En primer lugar, la $\mathrm{HC}$ está prácticamente centrada en los aspectos biológicos; sin embargo, apenas se hace alusión a cuestiones psicosociales. En salud mental esto tiene mayor relevancia, porque no solo se desea reparar un órgano, sino que la enfermedad mental conlleva sufrimiento psicológico y un empeoramiento en varias áreas: social, congnitiva, etc., pero que, además, abordarlas puede suscitar problemas morales. Todos estos aspectos deben ser tratados por diversos profesionales, los cuales han de reflexionar sobre cómo, por qué y de qué manera la persona ha de tener un tratamiento, sea biológico y/o psicológico. Por tanto, la HC no puede anclarse en lo meramente biológico, sino que se ha de estructurar y componer mediante aspectos biopsicosociales. 
En segundo lugar, en referencia a las anotaciones subjetivas, pese a que sí se contemplan en la ley $41 / 2002$, este cuerpo legal no da ningún tipo de definición. Algunas comunidades autónomas incorporan definiciones en sus propias regulaciones. Pensamos que sería conveniente una restructuración del artículo 18.3 de la ley 41/2002, con la finalidad de especificar a qué se refiere por "anotaciones subjetivas".

En tercer término, es necesario dejar constancia de algunas cuestiones éticas que puedan derivarse de la praxis médica. En psiquiatría hemos detectado algunos escenarios que pueden suscitar problemas morales, de modo que es conveniente dejar constancia de estos en la HC. Es necesario anotar toda información que se considere, desde un criterio médico, trascendental para el conocimiento veraz y actualizado del estado de salud del paciente. Estas anotaciones pueden ayudar a mejorar la relación asistencial, pero hay que tener especial cuidado con aquellos datos sensibles.

En cuarto lugar, tanto en la elaboración de la HC como en el manejo de la información la confidencialidad tiene un papel crucial. Para que haya un respeto por la persona durante la elaboración y manejo de la HC, son necesarios principios deontológicos. Lo normal será mantener la confidencialidad de los datos sanitarios; sin embargo, observamos que por definición la confidencialidad se presenta como prima facie, tal y como la concibe la normativa jurídica. Tanto en el respeto por la confidencialidad como para las excepciones que justifican su divulgación es preciso tener como eje central unos principios éticos. Pero para que haya un mayor respeto por dichos principios se requieren varios aspectos: 1) un mayor análisis y una ampliación de las normas legales que regulen la información sanitaria; 2) un aumento en el blindaje de los datos que asegure su confidencia- lidad, y 3) que los profesionales reflejen virtudes éticas que garanticen su compromiso, tanto con el paciente como hacia su propia profesión.

En quinto lugar, en cuanto al acceso a los datos sanitarios, pese a que varias personas pueden tener acceso, ha de haber un respeto por la intimidad de los implicados. Y es que la información puede referirse al propio paciente, a terceras personas o a los profesionales. Si el paciente u otras personas solicitan acceder a la HC, siempre y cuando esté justificado, no se entregará información confidencial sobre terceras personas o sobre las anotaciones subjetivas del profesional.

Por último, constatamos la poca investigación sobre la opinión de los implicados en la HC. Es preciso conocer cuáles son las percepciones de los pacientes y los profesionales al respecto. Los estudios llegan a la conclusión de que la confidencialidad tiene un papel crucial en la HC. En salud mental los pacientes están preocupados porque otros profesionales conozcan información sensible referente a su psicopatología, de modo que algunos piden que no se anote cierta información o que las borren de sus historiales. Nuevamente, esto indica la necesidad de establecer mecanismos informáticos de acceso para que haya un mayor respeto por la intimidad de los pacientes.

Varias son las cuestiones que este artículo no aborda o no resuelve satisfactoriamente: 1 ) ¿cómo diferenciar anotaciones subjetivas de las que son propiamente notas referentes al tratamiento y/o psicoterapia?; 2) ¡cómo establecer mecanismos informáticos que aseguren el respeto por la confidencialidad de los datos?; 3) ¿̨a quién pertenece la HC?, y 4) la exposición que realizamos sobre la opinión de los pacientes y los profesionales no aporta mucha evidencia empírica, lo cual podría tener sesgos al extraer conclusiones. 


\section{Referencias}

1. Ennis L, Rose D, Callard F, et al. Rapid Progress or Lenghty Process? Electronic Personal Health Records in Mental Health. BMC Psychiatry 2011; 11: 117.

2. Baca E. Teoría del sintoma mental. Madrid: Triacastela; 2007.

3. Abel F, Amarilla M, Boizas Mà, et al. Confidencialidad en la práctica psiquiátrica. Guía breve. Madrid: Asociación Española de Derecho Farmacéutico; 2002.

4. Gracia D. Procedimientos de decisión en ética clínica. Madrid: Triacastela; 2007.

5. Broggi M, Mejón R. Las “anotaciones subjetivas” en la historia clínica. Med Clin (Barc) 2004; 112(7): 279.

6. Ramos S. La toma de decisiones compartidas en pacientes con esquizofrenia: cuestiones médicas y éticas. Dilemata. Revista Internacional de Éticas Aplicadas 2012; 10: 263-277.

7. Ramos S. Las decisiones subrogadas en pacientes con esquizofrenia. Revista de Bioètica i dret 2015; 33: 68-81.

8. Ramos S. El consentimiento informado en la esquizofrenia: revisión del concepto “dignidad”. En: Boladeras M, (ed.) ¿Qué dignidad? Filosofía, Derecho y práctica sanitaria. Capellades; Proteus: 2010: 217-230.

9. Ramos S, Román B. Las voluntades anticipadas en pacientes con esquizofrenia: un instrumento para potenciar la autonomía. Revista de la Asociación Española de Neuropsiquiatría 2014; 34(121): 21-35.

10. Ramos S. La calidad de vida en pacientes con esquizofrenia y sus familias. Análisis bioético, conceptual y psicopatológico. Eidón. Revista de la Fundación Ciencias de la Salud 2014; 42: 46-56.

11. Broggi M. Gestión de los valores “ocultos” en la relación clínica. Med Clin (Barc) 2003; 121(18): 705-709.

12. Buchanan A, Brock D. Deciding for Others. The Ethics of Surrogate Decision Making. New York: Cambridge University Press; 1989.

13. Grupo de trabajo de bioética de la semFYC. Declaración de la Sociedad Española de Medicina de Familia y Comunitaria. Informatización y confidencialidad de la historia clínica. Aten Primaria 2004; 34(3): 140-142.

14. Beauchamp T, Childress J. Principios de ética biomédica. Barcelona: Masson; 1999.

15. Júdez J, Nicolás $P$, Delgado $\mathrm{M}^{\mathrm{a}}$, et al. La confidencialidad en la práctica clínica: historia clínica y gestión de la información. Med Clin (Barc) 2002; 118(1): 18-37.

16. Gracia D. Como arqueros al blanco. San Sebastián: Triacastela; 2004.

17. Fernández J. Régimen jurídico general de la historia clínica. En: Fernández J. La historia clínica. Granada: Comares; 2002.

18. Borrás M, Bursquets JM, García A. Proyecto "Historia Clínica Compartida” en Cataluña: marco legal y ejercicio de los derechos de acceso, rectificación, cancelación y oposición (ARCO). Med Clin (Barc) 2010; 134(supl 1) :16-20.

19. Hernando P, Seoane J, Francisco de Asís J. La reserva de las anotaciones subjetivas: ¿¿derecho o privilegio? Rev Calidad Asistencial 2006; 21(1): 31-38.

20. Comité de Bioètica de Catalunya. Recomanacions del Comité de Bioètica de Catalunya sobre la confidencialitat en la documentació clínica informatitzada. Disponible en: http://comitebioetica.cat/wp-content/uploads/2014/03/Confidencialitat-de-la-Informació-clinica-Informatitzada.pdf Consultada el 8 de enero de 2015.

21. Perera G, Holbrook A, Thabane L, et al. Views on Health Information Sharing and Privacy from Primary Care Practices Using Electronic Medical Records. International Journal of Medical Informatics 2011; 80: 94-101.

22. Salomon R, Urbano J, Rosenbloom S, et al. Openness of Patients' Reporting with Use of Electronic Records: Psychiatric Clinicians's view. J Am Med Inform Assoc 2009; 17: 54-60.

23. Fidel S, Eiroa F, Giannoni A, et al. Confidencialidad en las historias clínicas informatizadas en salud mental. Med Clin (Barc) 2013; 14(1): 30-37.

24. Iraburu M, Chamorro J, De Pedro M. Conocimientos, comportamientos y opiniones de los profesionales sanitarios de un hospital en relación a la confidencialidad. An Sist Sanit Navar 2006; 29(3): 357-366.

25. Boyer L, Renaud M, Limousin S, et al. Perception and use of an electronic medical record system by professionals of a public psychiatric hospital. L'Encéphale 2009; 35: 454-460.

26. Boyer L, Baumstrarck K, Belzeaux R, et al. Validation of a Professionals' Satisfaction Questionnaire with Electronic Medical Records (PSQ-EMR) in Psychiatry. European Psychiatry 2011; 26: 78-84.

Recibido: 31 de enero de 2015

Aceptado: 8 de mayo de 2015 